SRL-9-F-2004

\author{
Long-Lifetime Low-Scatter \\ Neutron Polarization Target
}

Prepared by

Dr. Jonathan M. Richardson

Science Research Laboratory

15 Ward Street

Somerville, MA 02143

Prepared for

Department of Energy

Phase I Final Technical Report

Period for July 21, 2003 to April 20, 2004

Contract No. DEFG02-03ER83771

July 9, 2004 use of these data by third parties. This notice shall be affixed to any reproductions of these data in whole or in part.” 


\title{
Long-Lifetime Low-Scatter Neutron Polarization Target
}

\author{
DOE Phase I Final Report \\ Science Research Laboratory, Inc. \\ Jonathan M. Richardson, Ph.D.
}

\section{Table of Contents}

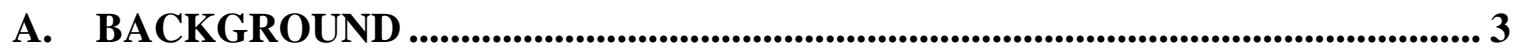

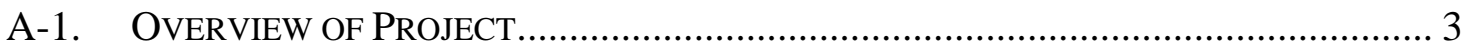

A-2. POLARIZED ${ }^{3} \mathrm{HE}$ AS A NEUTRON POLARIZER ……............................................ 5

A-3. POLARIZED ${ }^{3}$ He tARgETS AS A NEUTRON POLARIZATION ANALYZERS ................. 7

A-4. MAKING USEFUL ${ }^{3}$ He TARgETS ........................................................................ 8

A-5. THE SRL ${ }^{3}$ He PolarizATION SYSTEM ……................................................... 10

B. PHASE I ACCOMPLISHMENTS......................................................................... 12

B-1. PHASE I OBJECTIVES............................................................................... 12

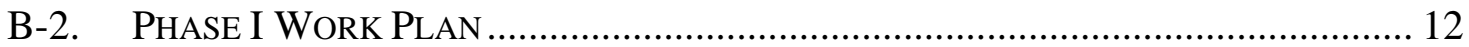

B-2-a. Fabrication of test cell ...................................................................... 12

B-2-b. Polarization Lifetime Measurements ....................................................... 15

C. LITTERATURE CITED............................................................................................ 17 


\section{A. Background}

\section{A-1. Overview of Project}

The goal of this Phase I project was to make improved ${ }^{3}$ He neutron spin filters for polarized neutron scattering experiments. We have accomplished this goal, although the testing of these targets is ongoing. Following this project in Phase II, we will collaborate with instrument scientists at the planned Spallation Neutron Source and elsewhere to implement our filter technology in one or more instruments. Ultimately, we envision a Phase III program where we supply ${ }^{3} \mathrm{He}$ spin filter technologies to the various neutron scattering facilities throughout the world. This program builds on ${ }^{3} \mathrm{He}$ polarization projects completed and currently underway at SRL.

Neutron scattering is a well-established tool for the investigation of surface and bulk structure of magnetic and non-magnetic materials, for the characterization of polymers, crystals, and superconductors, and for numerous other applications in materials science and analytical chemistry. There are 8 neutron scattering centers in the US and Canada and over 22 abroad. Unlike other methods (such as x-ray scattering), neutrons penetrate deeply into most materials and can distinguish among different isotopes of low-Z atoms including hydrogen. Neutron scattering instruments include small angle spectrometers (SANS) that probe bulk structure, reflectometers that probe surfaces and films, defractometers that characterize crystals, and numerous other instruments for characterizing the structure and composition of materials.

Neutrons are uncharged, spin-1/2 particles that interact with both magnetic and nonmagnetic materials in a spin-dependent way. In the case of non-magnetic materials, this fact can be exploited in order to distinguish coherent scattering (which gives information about material structure) from the ever-present background of incoherently scattered neutrons. In the case of magnetic materials, the use of polarized neutrons distinguishes between magnetic and non-magnetic scattering. Polarized neutron scattering has been demonstrated to be a very powerful tool in studies of deuterium-tagged polymers [1, 2] and has led to its use in the study of biological macro-molecules [3-6]. Another important application of polarized neutron scattering is in the study of magnetic materials including magnetic surfaces [7], ferromagnets [8], and superconductors [9, 10]. Most polarizedneutron scattering experiments to date have been performed without the aid of spin analysis. Knowledge of the spin of the exit neutron greatly improves the ability to distinguish between coherent and incoherent neutron scattering [11].

Thermal and cold neutrons (those with energies less than $25 \mathrm{meV}$ ) are most commonly polarized by spin-dependent grazing-incidence reflection from thin, magnetically polarized films [12]. Present state-of-the-art multi-layer thin films ("supermirrors") [13] offer the highest spin-dependent critical angles, which are, in any case, only a few milliradians. There are several major drawbacks to this technique. Most importantly, the angular acceptance of supermirrors polarizers is limited by the critical angle so that it is problematic to use a supermirror as a spin analyzer in many instruments. Second, supermirrors cannot be used to polarize epithermal neutrons (those with energies higher than $25 \mathrm{meV}$ ). Third, when polarizing a beam using a supermirror, the unwanted neutrons are scattered out at small angles relative to the primary beam. Supermirror assemblies 
must be carefully shielded in order to remove gamma radiation created from unavoidable interactions of the unwanted neutron beam with surrounding materials.

It has been demonstrated that thermal neutrons can be polarized by passing them through a dense ( 1 atmosphere), spin-polarized, gaseous ${ }^{3}$ He target [14]. Because the neutron absorption cross section of polarized ${ }^{3} \mathrm{He}$ is strongly spin-dependent, a sufficiently thick polarized target transmits neutrons that are in one spin state and strongly attenuates the other. A ${ }^{3} \mathrm{He}$ target can be made such that the absorption is essentially angle-independent allowing for polarization of highly divergent beams such as occur between a sample and detector. The absorbed neutrons create primarily charged particles that are easily shielded. Unlike supermirror reflection, a dense ${ }^{3} \mathrm{He}$ filter can be used to polarize epithermal neutrons [15], which have a number of important applications [16]. Polarized ${ }^{3} \mathrm{He}$ can be used as a polarization analyzer in a number of important neutron scattering instruments, including Small Angle Neutron Scattering (SANS) spectrometers [11].

One difficulty in developing ${ }^{3}$ He neutron spin filters is that they must often take complex shapes that are not easily made from the most widely used material: aluminosilicate glass. Aluminosilicate glass, e.g. GE-180, is both relatively transparent to neutrons and has a long hold time for ${ }^{3} \mathrm{He}$ polarization. It suffers considerably, however, from availability and workability. We propose to develop spin filters from specially prepared and coated aluminum, which has been shown to have a long storage lifetime for ${ }^{3}$ He even in its raw state. One group has observed lifetimes of up to 10 hours in raw aluminum containers [17]. This proposal describes coating methods that should be able to increase this lifetime to 40 hours or more. Aluminum can be formed into complex shapes with flat or curved faces and is highly transparent to neutrons.

Over the past several years, SRL has been in contact with a number of researchers who are interested in using ${ }^{3} \mathrm{He}$ neutron polarizer/analyzers to study magnetic and other materials. Some of the institutions include the IBM Almaden Research Center, Seagate, the National Institute of Standards and Technology Center for Neutron Research (NCNR), the Argonne National Laboratory (ANL) Intense Pulsed Neutron Source (IPNS), and the planned Spallation Neutron Source (SNS), which is scheduled for completion in June 2006. Previously, SRL participated in a demonstration of polarized neutron scattering at the IPNS that utilized a neutron spin filter to analyze the neutron beam. This was the first demonstration of polarized neutron reflectometery using a ${ }^{3} \mathrm{He}$ neutron spin analyzer to be performed in the US. In this experiment, the spin filter was filled with polarized ${ }^{3} \mathrm{He}$ by our collaborators at the Indiana University and transported by car to the IPNS where it was installed on the POSY neutron reflectometer. More such collaborations are planned during the Phase II portion of this project using the targets described in this proposal.

A specific and compelling application of polarized neutron scattering is the characterization of magnetic properties of materials used in magnetic disk drive surfaces and read heads. This application has been suggested by researchers at IBM's Almaden Research Center [18]. We have also contacted Dr. Christina Hoffmann, who is an instrument scientist for the Spallation Neutron Source (SNS). Dr. Hoffman is heading the effort to design a polarized neutron single crystal diffractometer that will operate on a thermal beamline at the SNS. The instrument will be used to study the magnetic structure 
of crystals. This instrument produces highly divergent scattered beams that can only be spin-analyzed using ${ }^{3} \mathrm{He}$. It also will use epithermal neutrons that are not easily initially polarized except by using a ${ }^{3} \mathrm{He}$ spin filter, as shown in the next section.

Current work at Science Research Laboratory has resulted in the development of a ${ }^{3} \mathrm{He}$ polarization system that is still under final testing. This system should be fully operational in the next few months and will be used in the propose program. Several of the technologies that have been incorporated in SRL's polarizer will also be used to make the novel spin filters discussed in this proposal, including SRL's non-magnetic valve technology.

\section{A-2. Polarized ${ }^{3} \mathrm{He}$ as a neutron polarizer}

Highly polarized gaseous ${ }^{3} \mathrm{He}$ is an ideal medium for polarizing thermal, cold, and epithermal neutrons because of the large spin dependence of the ${ }^{3} \mathrm{He}(\mathrm{n}, \alpha)$ cross section. The thermal neutron absorption cross section is $\sigma^{\uparrow \downarrow}=10658 \pm 30 \mathrm{~b}$ for the anti-parallel case and $\sigma^{\uparrow \uparrow}=2 \mathrm{~b}$ for the parallel case [19]. The performance of the polarizer is determined by the linear density ( $\rho \ell$, the product of the density and length along the beam) and by the degree of ${ }^{3}$ He polarization $-1 \leq P_{T} \leq 1$, defined by

$$
P_{T} \equiv \frac{\rho^{\uparrow}-\rho^{\downarrow}}{\rho},
$$

where $\rho^{\uparrow(\downarrow)}$ is the number density of spin up (down) of the ${ }^{3} \mathrm{He}$ and the total density is $\rho=\rho^{\uparrow}+\rho^{\downarrow}$. Upon passage through the polarized gas, a previously unpolarized thermal neutron beam acquires a polarization given by

$$
P_{n} \equiv \frac{t^{\uparrow}-t^{\downarrow}}{t} \approx \frac{1-\exp \left(-\sigma^{\uparrow \downarrow} \rho \ell P\right)_{t}}{1+\exp \left(-\sigma^{\uparrow \downarrow} \rho \ell P_{t}\right)}=\tanh \left(\sigma^{\uparrow \downarrow} \rho \ell P / 2\right),
$$

where $t^{\uparrow(\downarrow)}$ is the transmission of neutrons with spin up (down), $t=t^{\uparrow}+t^{\downarrow}$ is the total transmission, and absorption of the parallel state has been ignored. If the particular application demands high neutron polarization (at the expense of transmission), this can be provided simply by increasing the linear density of the target. Often, however, the precision of a measurement in a particular time-period is proportional to a figure of merit $F \equiv P_{n} \sqrt{t}$, which is calculated under a number of different conditions in Figures 1 and 2.

As shown in Figures 1 and 2, the performance of a Polarized ${ }^{3} \mathrm{He}$ spin filter depends both on the ${ }^{3}$ He polarization and the effective thickness $\rho \ell$, which can be varied by changing the pressure and/or thickness of the filter. For less-than-perfect ${ }^{3} \mathrm{He}$ polarizations, there is a trade-off between high polarization of the transmitted neutron beam or high transmission overall. Figure 1 shows a calculation of the figure of merit $\mathrm{F}$ as a function of $P_{n}$ and $\rho \ell$ for both thermal and epithermal neutrons. From these curves it should be clear that, in the case of neutrons with energies below 25 meV (mili-electron volts), the ${ }^{3}$ He polarization must be higher than $90 \%$ in order for a ${ }^{3} \mathrm{He}$ polarizer to be a viable 
alternative to a grazing-incidence polarizer, assuming a well collimated beam. Even if ${ }^{3} \mathrm{He}$ spin filters with $90 \%{ }^{3} \mathrm{He}$ polarization become available, they offer few advantages over grazing incidence polarizers in the case of highly collimated beams. In the case of epithermal neutrons, ${ }^{3}$ He spin filters are very competitive with alternative polarization schemes, even given relatively modest ${ }^{3} \mathrm{He}$ polarizations ( $\left.50 \%\right)$. At neutron energies above $20 \mathrm{meV}$, few materials offer significant spin absorption asymmetry and grazingincidence polarizers become ineffective. The use of ${ }^{3} \mathrm{He}$ spin filters as spin analyzers is discussed in the next section.

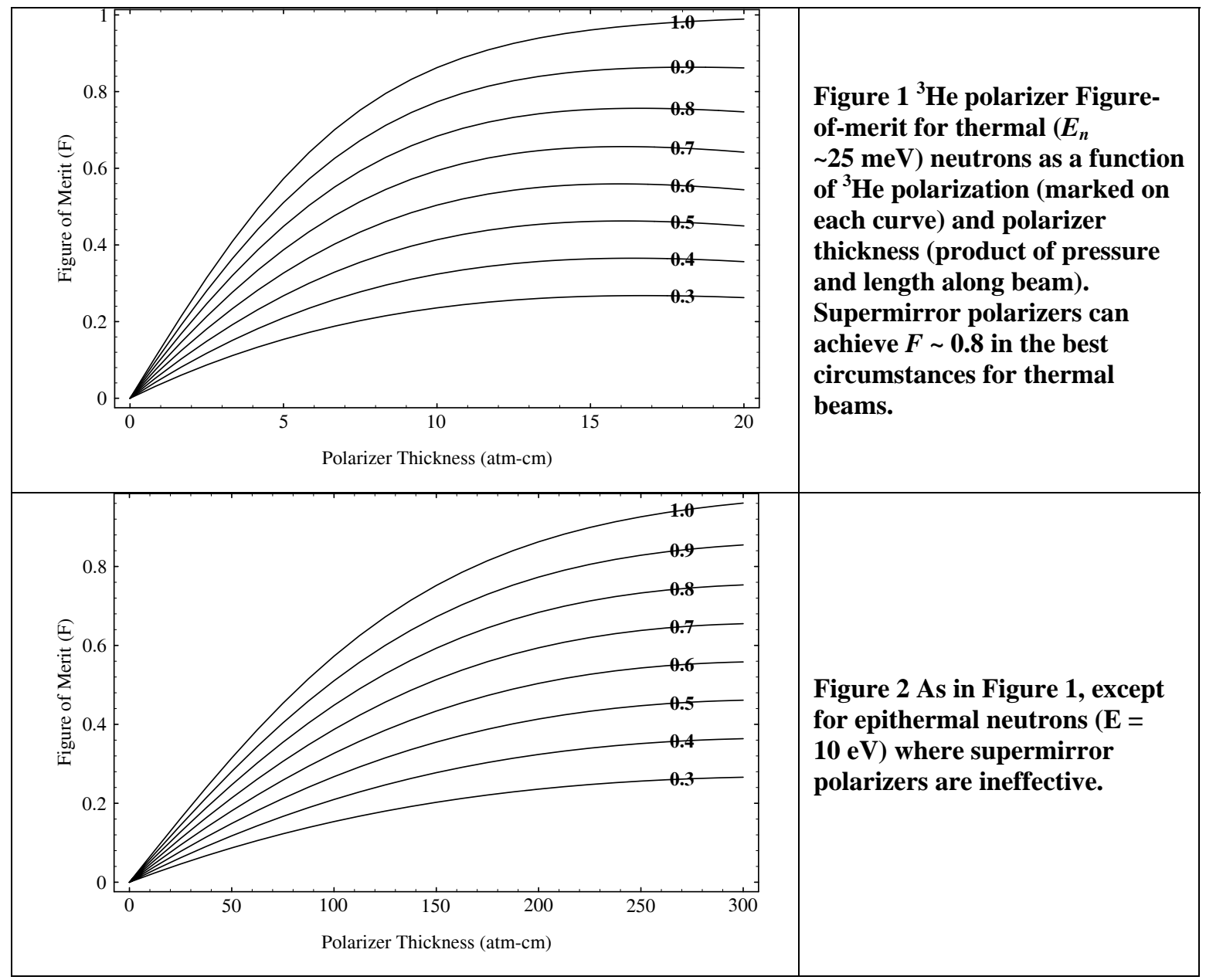

One important feature of the ${ }^{3} \mathrm{He}$ spin filter is that the polarization of the transmitted beam can be determined without precise knowledge of the ${ }^{3} \mathrm{He}$ polarization [14, 20]. This is accomplished by measuring the filter transmission when the ${ }^{3} \mathrm{He}$ is unpolarized $\left(t_{0}\right)$ and when it is polarized $(t)$. From these two measurements, it can be shown that, for an initially unpolarized beam, the neutron polarization of the transmitted beam is given by

$$
P_{n}=\sqrt{1-t_{0}^{2} / t^{2}}
$$


By measuring $t_{0}$ in advance, this procedure allows for continuous determination of the induced beam polarization regardless of the polarization of the ${ }^{3} \mathrm{He}$ within the filter.

\section{A-3. Polarized ${ }^{3}$ He targets as a Neutron Polarization Analyzers}

There is considerable interest in using a ${ }^{3} \mathrm{He}$ spin filter as an spin analyzer by placing it between a sample and detector in a manner shown in Figure 3 [21]. In this configuration, the polarized ${ }^{3}$ He discriminates between scatter that involves a spin flip from scatter that does not. Such a measurement will be sensitive to both magnetic and non-magnetic structure of the sample, and can be used to determine many critical parameters including magnetic domain size and orientation. Experiments of this type can also distinguish between coherent and incoherent neutron scattering from non-magnetic samples and thus offer higher sensitivity to material structure and composition.

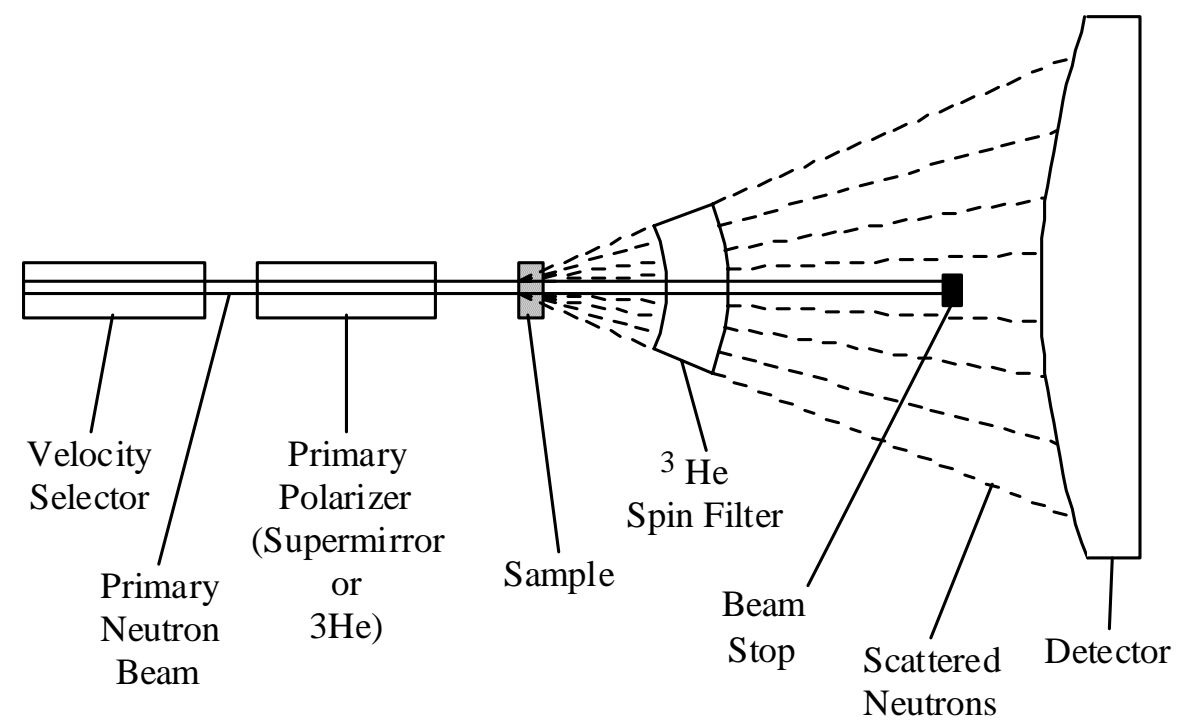

Figure 3 Example of a polarized small angle neutron scattering (SANS) experiment where the scattered beam is spin-analyzed. Such an experiment could be used to study magnetic materials.

In order to compare ${ }^{3} \mathrm{He}$ spin analyzers with existing supermirror spin analyzers, it is first important to understand that the goal is typically both to determine the angular dependence of the scattering cross section while also determining the spin dependence of the cross section. Cussen et al. [22] have shown that a measurement to determine the spin-dependent scattering cross section at narrow angular range will take 2.7 times as longer using an optimized ${ }^{3}$ He spin filter with $P_{n}=50 \%$ than can be achieved using a supermirror. Now consider the fact that the scattered beam may have an extended angular range, perhaps $360^{\circ}$. A supermirror analyzer might be scanned across the angular range in steps of its acceptance, which increases linearly with wavelength. This would require hundreds or thousands of steps, depending on the wavelength, which is part of the reason it is not a common practice. One alternative is to implement a supermirror array capable 
of simultaneously analyzing a broad range of angles. Such a device is expensive, difficult to manufacture, and loses a large fraction of the signal due to absorption.

The ideal thickness for a ${ }^{3} \mathrm{He}$ neutron analyzer depends on the wavelength of the neutrons being analyzed as well as the ${ }^{3} \mathrm{He}$ polarization. In the case of $50 \%{ }^{3} \mathrm{He}$ polarization, the ideal thickness for thermal neutrons (wavelength $\bar{\lambda} \approx 2.35 \AA$ ) is $22.5 \mathrm{~atm}-\mathrm{cm}$. Depending on the neutron scattering experiment being conducted (SANS, reflectometery, diffractometry, etc.), the spin filter could have 2-10 atm-liters of volume. Filling these relatively large volumes with polarized gas at high ( $>50 \%$ ) polarization will require a world-class ${ }^{3} \mathrm{He}$ polarization system such as the one under development at SRL.

\section{A-4. Making Useful ${ }^{3} \mathrm{He}$ Targets}

Making a useful ${ }^{3} \mathrm{He}$ target is complex due to the few materials that are both nonabsorbing for neutrons and low depolarizing for ${ }^{3} \mathrm{He}$. Filters with volumes of 2 liters or more will require one or more hours to fill. It would be inconvenient if they could not operate for at least several hours before recharging. The material that has been exploited by most groups is aluminosilicate glass (e.g., Corning 1720 and GE-180), from which manifolds with up to $100 \mathrm{~h}{ }^{3} \mathrm{He}$ polarization lifetimes have been made [23, 24]. For reasons that are only recently becoming clear, certain glass manifolds have a very low depolarizating effect on ${ }^{3} \mathrm{He}$. The difficulty is that aluminosilicate glass can only be worked in a narrow temperature range and is only commercially available in limited sizes (primarily small diameter tubing for in GE-180 only). Borosilicate glass (Pyrex) is not acceptable as a neutron spin filter due to high neutron absorption in boron. Making complex manifolds from aluminosilicate glass is difficult and expensive. It is not clear that large (> 2 liter) shapes can be manufactured with high geometrical tolerance at all.

The limitations of glassblowing have limited the geometries of the targets to relatively simple shapes thus far. There are several desirable shapes for neutron polarizers including that shown in Figure 4. It is desirable for these shapes to have relatively well defined geometries so that the absorption is not strongly dependent on angle.

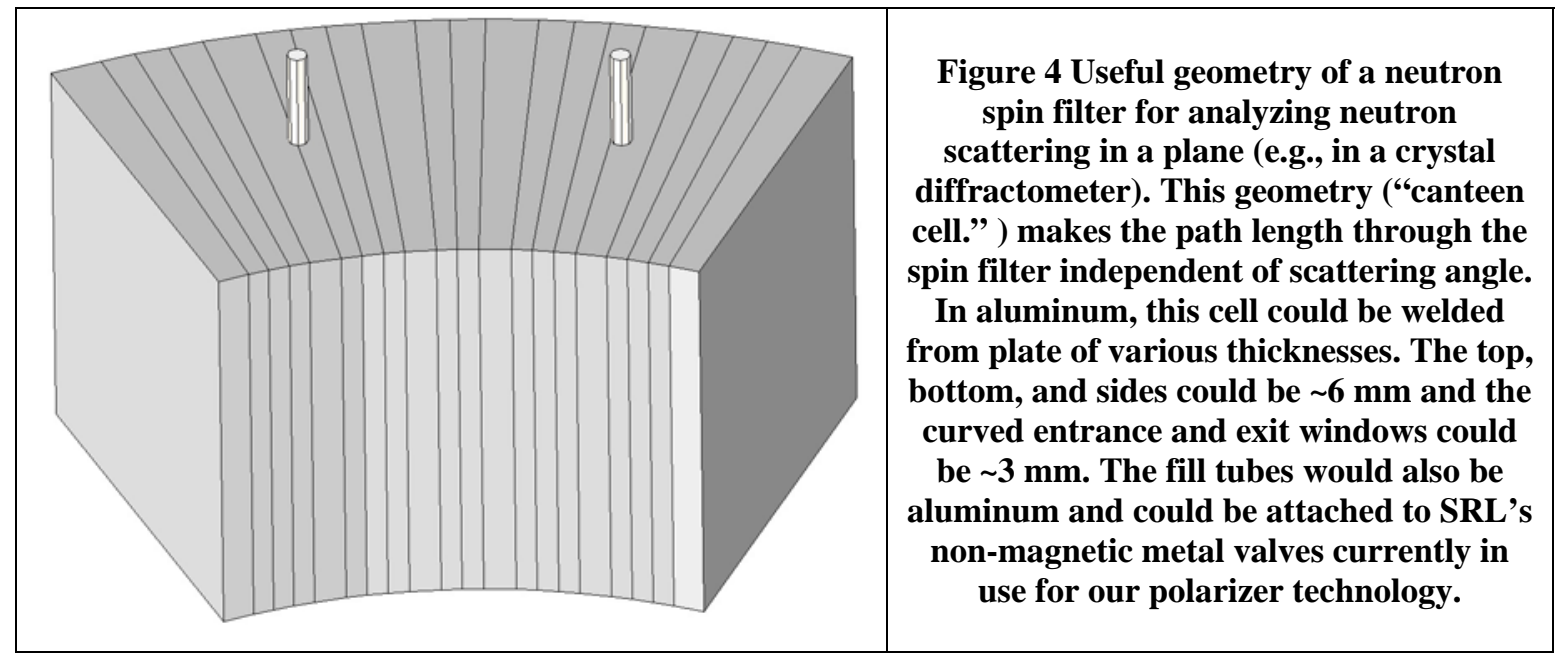

In this proposal we suggest that a good choice for refillable neutron spin filters would be aluminum, since this material has very low neutron absorption and, in its raw form, has been shown to have a relatively low depolarizing effect on ${ }^{3} \mathrm{He}$ [25]. In one measurement, 
lifetimes of up to 10 hours have been observed in raw aluminum cells [17]. With suitable internal coating, there is good reason to believe that the depolarizing effect can be reduced further, allowing the manufacture of large complex manifolds with ${ }^{3} \mathrm{He}$ hold times rivaling that of glass manifolds. When handled properly, aluminum has by far the lowest outgassing rate of any known material, including glass and steel [26] so that it will not poison the ${ }^{3} \mathrm{He}$ sample. In addition, aluminum is not porous to helium as is glass.

Complex aluminum manifolds could be made from aluminum sheet of various thicknesses, using thin $(\sim 3 \mathrm{~mm})$ plate wherever the neutron beam will impinge. All seams will be welded to high vacuum standards. SRL has already contacted a machine shop that specializes in the manufacture of aluminum ultrahigh vacuum chambers, and has received a rough quotation for the cell shown in Figure 4 of a few hundred US dollars. Another key technology will be the valves use to seal the manifold once filled. SRL has developed non-magnetic non-elastomer bellows valves as part of its ${ }^{3} \mathrm{He}$ polarizer program. These valves are made primarily from phosphor bronze and seal with either copper or Teflon. They may be welded directly to the fill tubes to avoid possible contamination.

The exact depolarization rate due to interactions with the manifold will depend on the alloy used as well as surface preparation. Pure aluminum (1100) is widely available and can be machined and welded, however it is much weaker than when alloyed. Two common alloys are 6061 , which contains $0.6 \%$ silicon, $0.28 \%$ copper, $1 \%$ magnesium, and $0.2 \%$ chromium; and 6063 , which contains $0.5 \%$ magnesium, and $0.5 \%$ silicon. Of the two, 6062 is considerably stronger, however it contains a small amount of chromium, which has a magnetic susceptibility that is 10 times that of any other additive. Both alloys are commonly available and can be machined, bent, and/or welded into complex shapes.

Bare aluminum has been shown to yield ${ }^{3} \mathrm{He}$ polarization lifetimes greater than 10 hours [17]. It is not known why the lifetimes are not even longer, however it may be that aluminum acquires ferromagnetic impurities on the surface while being formed. We suggest that much longer lifetimes are achievable by using appropriate coatings such as have been used to greatly decrease the depolarization effects of borosilicate glass [24]. In the case of borosilicate glass, the ${ }^{3} \mathrm{He}$ polarization lifetime was increased from 1 hour for uncoated cells to as much as 100 hours for cells that were internally coated with cesium.

Developing methods for preparing and coating the internal surfaces of aluminum manifolds are the primary focus of this Phase I and II program. Material preparation will be similar to the handling of ultrahigh vacuum equipment. The manifolds will be manufactured from polished stock to reduce surface roughness. The inner surfaces will be degreased and acid etched to remove any surface impurities. Finally, the manifold will be evacuated and baked at high temperature for many hours.

Coating with cesium poses some difficulties since cesium can never be exposed to air. There is also the distinct possibility that cesium will interact with the aluminum or its additives in an undesirable way. SRL has worked extensively with alkali materials and has an argon glove box for such purposes. The problem of interactions between cesium and the manifold can be avoided, if necessary, by precoating with an inert substance such as octadecyltrichlorosilane (OTS), which has been used by several groups to coat glass manifolds also exposed to alkalis [23]. 


\section{A-5. The SRL ${ }^{3}$ He Polarization System}

SRL and the principal investigator of this proposed project in particular have been involved with ${ }^{3} \mathrm{He}$ polarization for many years. Our first project concerned an effort to increase the pressure at which ${ }^{3} \mathrm{He}$ may be polarized using the direct method. Presently, we are working to build a ${ }^{3} \mathrm{He}$ polarizer that uses a compression stage. The focus of our program has been toward developing high-throughput ${ }^{3} \mathrm{He}$ polarization technologies for both neutron polarization and medical imaging. We also have been involved with lowfield NMR measurements of material microstructure [27].

Figure 5 shows SRL's ${ }^{3}$ He polarization system, which will soon be capable of producing 1 liter-atm per hour at a polarization of at least $50 \%$. Our system relies on optical pumping of the metastable species of ${ }^{3} \mathrm{He}$ [28] at low pressures ( 1 bar), where after the gas is compressed to 1-2 bars by a two-stage non-magnetic pumping system similar to that developed at University of Maintz in Germany [29, 30]. We have developed two compression technologies. One uses an aluminum piston with elastomer seals, similar to the proven Maintz design. This system requires the use of fluorinated grease to lubricate the seal. It is desirable to eliminate elastomers and grease since these materials have higher outgassing and ${ }^{3} \mathrm{He}$ depolarization rates than other materials. To this end, we have worked extensively with a dry pumping technology that uses a glass cylinder and Teflon seals. This method has not yet been shown to have the necessary performance and is still under development.

Our system includes a diode-pumped solid-state laser capable of producing in excess of $2 \mathrm{~W}$ narrow-band that is shown in Figure 7. This laser technology has proven to be highly reliable and trouble free in hours of use in our laboratory. SRL has made plans to scale this laser to greater than $5 \mathrm{~W}$ narrow-band in future projects.

In designing this system, we have attempted to utilize off-the-shelf technologies wherever possible. This has lowered the system cost and improved the reliability of many components. As part of this program, we have developed non-magnetic bellows-sealed valves that are incorporated in the pump head. The valve bodies are aluminum and the stem and bellows are phosphor bronze. The seal is made with Teflon or OHFC copper. These valves can ultimately be used in the all-metal neutron spin filters under development in this project.

Another important technology developed at SRL is an all-glass non-magnetic titanium getter used to maintain the purity of the ${ }^{3} \mathrm{He}$ as it passes through the compression system. The details of this technology are proprietary, however this subsystem is critical to the operation of the polarizer since impurities reduce the polarization achievable in the lowpressure optical pumping cell. 


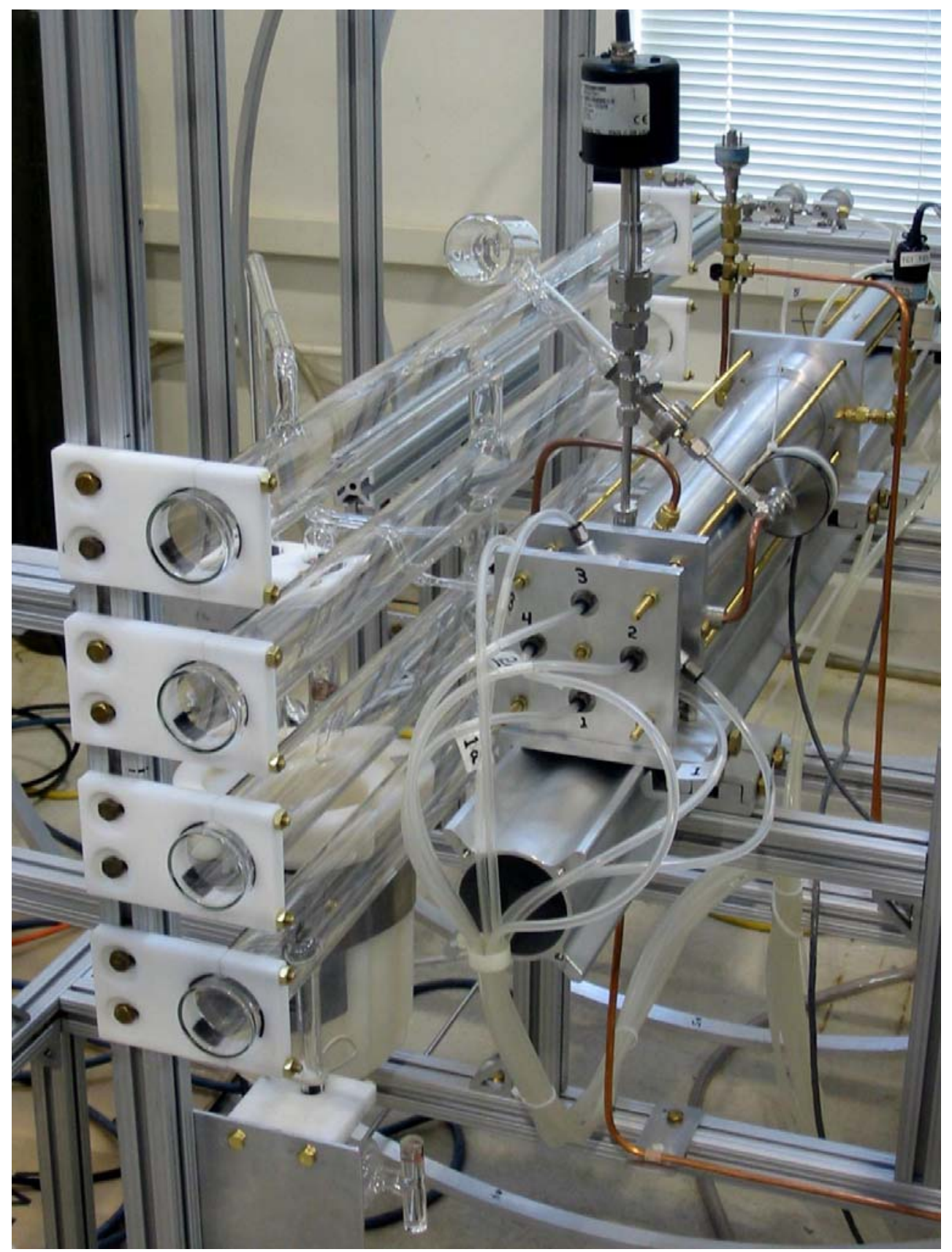

Figure 5 SRL's ${ }^{3}$ He polarization system.

Science Research Laboratory, Inc. 


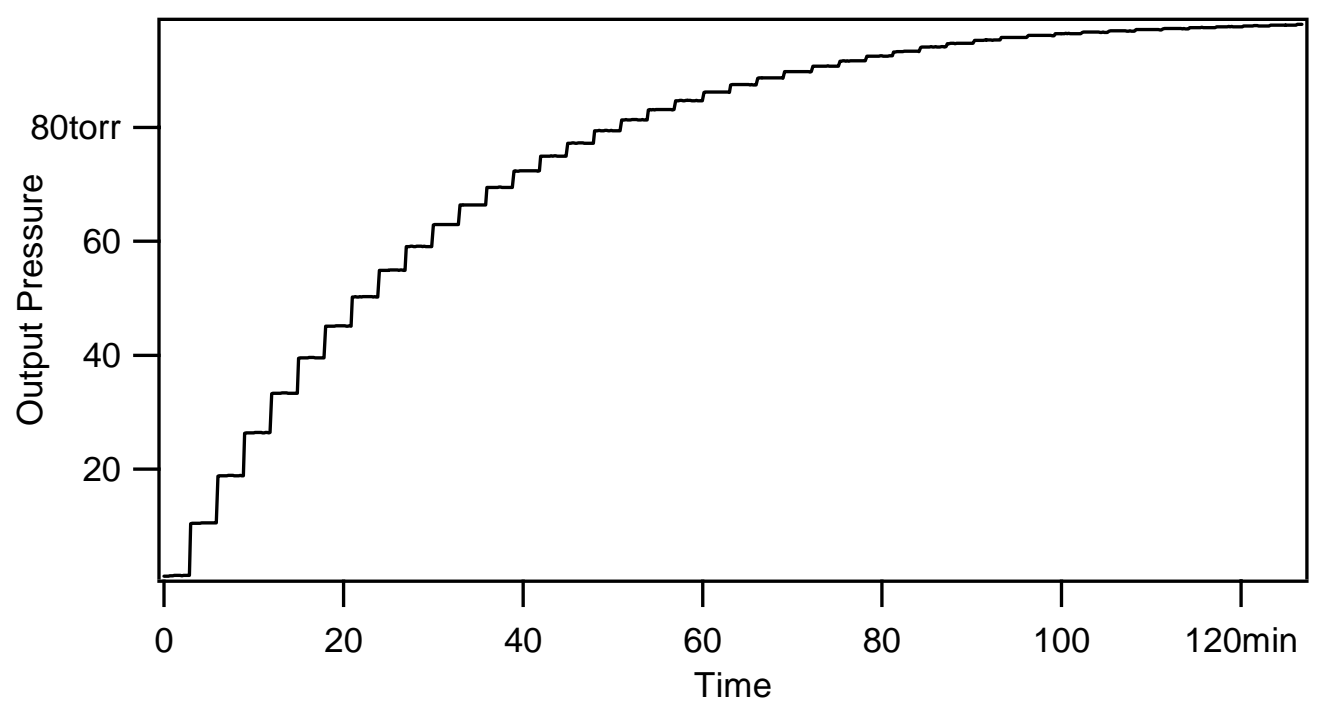

Figure 6: Results of tests of the compressor system. In this test, gas was pumped from the OP cells at approximately 1 torr into a primary storage volume.

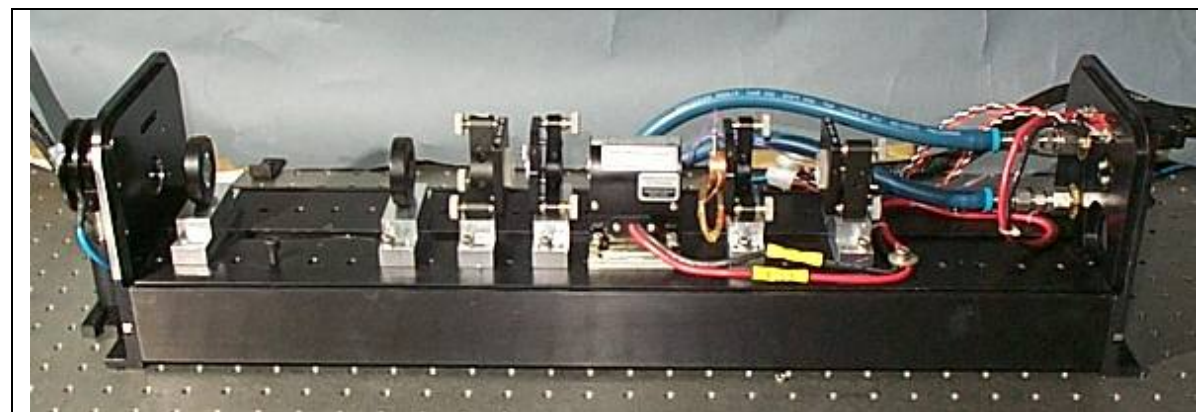

Figure 7 SRL's

diode pumped

Nd:LMA laser

system capable of

greater than $2 \mathrm{~W}$

output into a

narrow band.

\section{B. Phase I Accomplishments}

\section{B-1. Phase I Objectives}

- Fabrication of a test cell

- Polarization lifetime measurements

\section{B-2. Phase I Work Plan}

\section{B-2-a. Fabrication of test cell}

SRL has fabricated a cell that is largely glass with an aluminum sheet at one end. Using a combination of aluminum and glass will allows for ${ }^{3} \mathrm{He}$ polarization to be measured using SRL's low-field NMR as described in the next section. The seal between the aluminum and glass is indium. The cell is shown in Figure 8. This cell is undergoing cleaning via low-pressure helium discharge. By monitoring the atomic lines from the discharge, we can determine the purity of the sample and thus the cleanliness of the cell. 


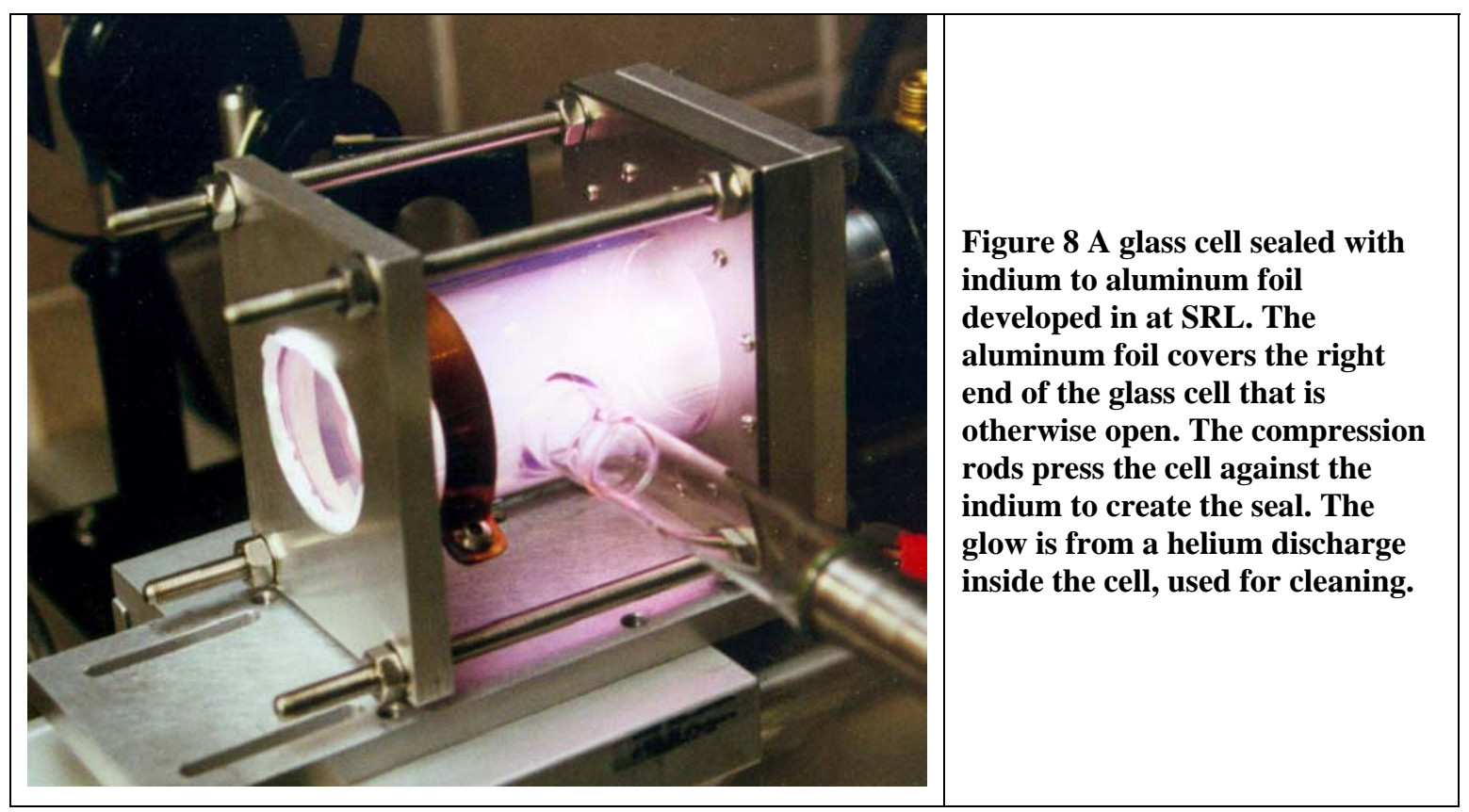

The method for assembly of these cells is as follows: Once the glassware is made and cleaned, the open end is polished flat and pressed against the aluminum sample with indium in between. The aluminum and glass are pre-baked in a vacuum oven to drive out impurities and then stored under dry nitrogen until use. We assembled the system with cleaned indium, etched the internal surfaces with "Piranha solution" (a mixture of hydrogen peroxide $\mathrm{H}_{2} \mathrm{O}_{2}$ and sulfuric acid $\mathrm{H}_{2} \mathrm{SO}_{4}$ ), rinsed with methanol and finally dionized water several times, and dried with a nitrogen jet. All chemical work was performed under a chemical hood with appropriate protective wear.

Glassware has been coated by other groups by using $2 \mathrm{mM}(0.788 \mathrm{cc} / \mathrm{liter})$ solution of OTS in $80 \%$ n-hexane plus $12 \% \mathrm{CCl}_{4}$ and $8 \% \mathrm{CHCl}_{3}$ by volume [33]. The volume should be filled with this solution for 5 minutes and then dried under nitrogen, cleaned with $\mathrm{CHCl}_{3}$, dried again, then evacuated and baked for many hours at $150^{\circ} \mathrm{C}$, which is the maximum safe temperature to bake the indium seal.

An important technology for making aluminum cells is the development of a suitable valve. SRL has developed a valve design based on a commercial valve insert that can be coupled to a custom aluminum valve body. The resulting technology is a valve that is non-magnetic, has low dead space, and requires no lubrication. The commercial valve insert is made by Swagelok (model B-4HK-KA), and is made from non-magnetic materials as shown in Figure 9 except for a spring that can be replaced with phosphor bronze or a similar material. The valve body seals to the insert using a Teflon-coated copper gasket. The valve seat is available in several different materials including copper. So far, we have used "Kel-F," which is a clear hard plastic. In all tests, the valves have performed well. 


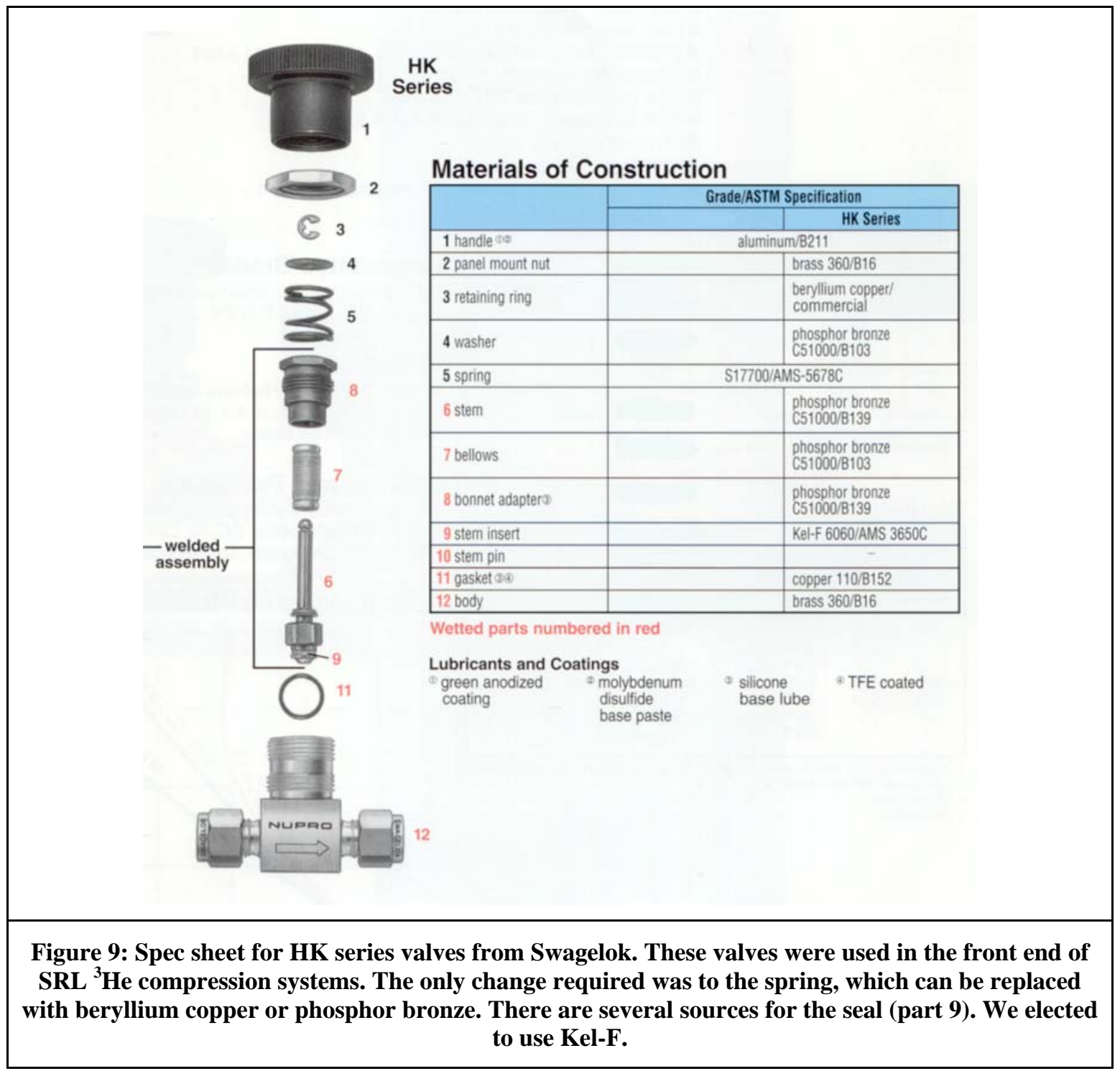

Coating with cesium can best be accomplished under vacuum by placing a sealed glass cesium ampoule inside an adjoining glass cell, breaking the seal with a sealed magnet, and distilling a small amount into the cell by heating ampoule with a torch. Once accomplished, the cell containing the ampoule will be removed with a torch and the end sealed off.

During this Phase I program we were able to assemble a test cell and found it to be vacuum-sealed, clean, and operational. We also tested the non-magnetic valve system and found it to work well. We were unable to perform the OTS or cesium coating with the available time constraints. This part of the project is ongoing and will become part of the Phase II program. 


\section{B-2-b. Polarization Lifetime Measurements}

The helium polarization can be directly monitored using low-field NMR [34]. SRL has built and tested the low-field NMR apparatus shown in Figure 10. A schematic of the apparatus is shown in Figure 11. This apparatus has been calibrated by measuring the signal from water, which acquires a known magnetization as a function of field strength. We have also measured the signal from a low-pressure helium discharge. It should be noted that calibration is not necessary for measurements of polarization lifetime.

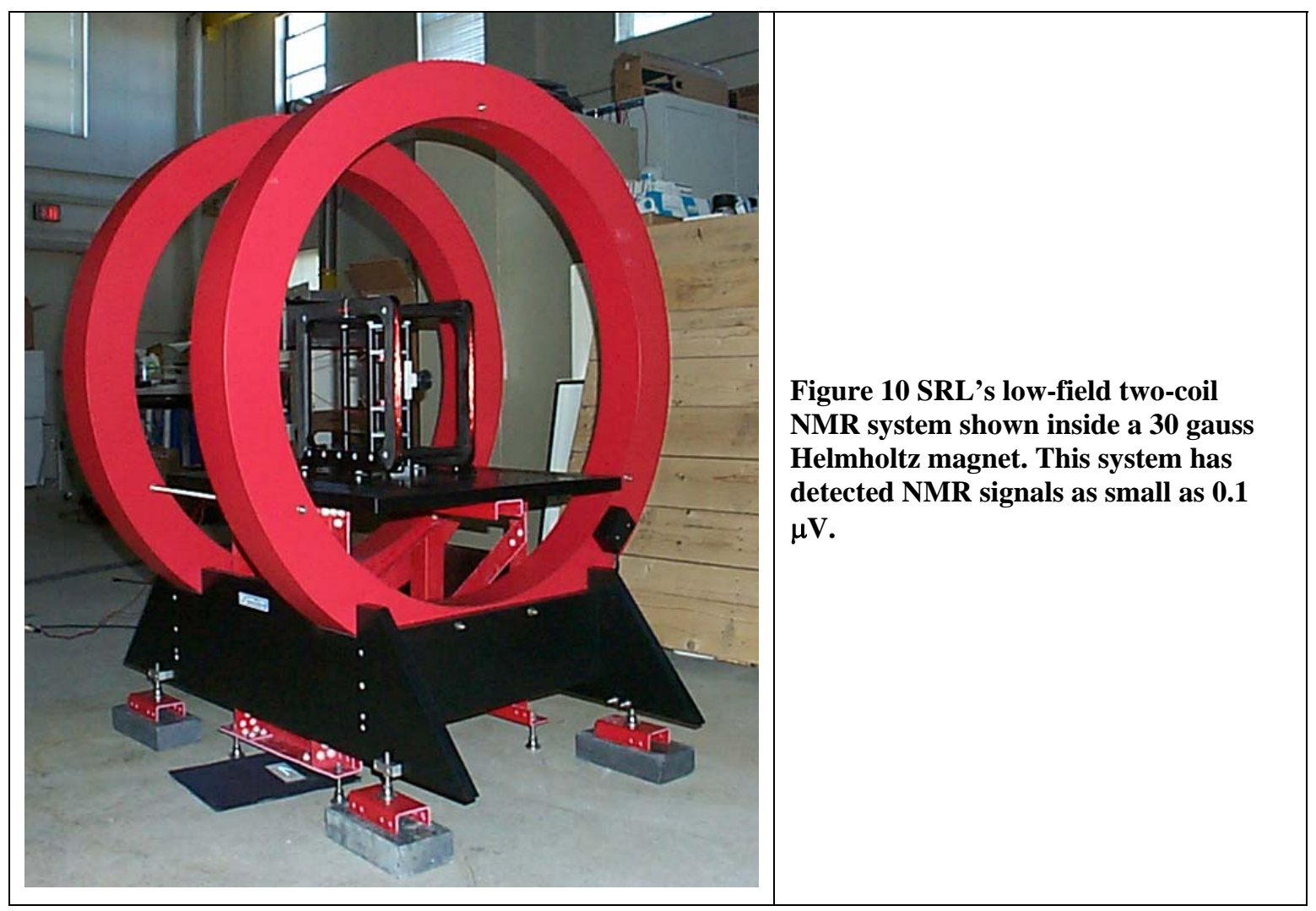

Measurement of the polarization lifetime is accomplished using a method called adiabatic fast passage (AFP) wherein the magnetization of the ${ }^{3} \mathrm{He}$ is flipped by scanning the primary field through resonance [34]. As the magnetization flips, it creates a signal proportional to its magnitude. Losses due to AFP itself (rather than interactions with the walls) is measured by flipping the magnetization repeatedly in a short period of time. Note that our magnet has been designed to have magnetic field gradients that are below $0.1 \%$ in the region of our cell so that losses due to gradient interactions will be minimized.

In AFP method, the excitation coils are continuously driven while the pickup coils are continuously monitored. At the same time, the main field strength is ramped such that the sample passes through resonance. Done correctly, this will flip the magnetization from parallel to anti-parallel or back with little loss of polarization. In order to sense the relatively small NMR signal, the relatively large signal that couples from the excitation 
coils into the pickup system must be removed or "nulled." This is accomplished in two steps. First, the pickup coils are oriented perpendicular to the excitation coils, minimizing pickup. The orientation was adjusted mechanically such that the coupling is minimized. It is not possible to completely zero the coupling by this method apparently due to a small amount of capacitive coupling. A drive coil was added close to the pickup system that was fed a signal chosen such as to cancel the capacitive signal. In this way, the system could be nulled to below a few microvolts.

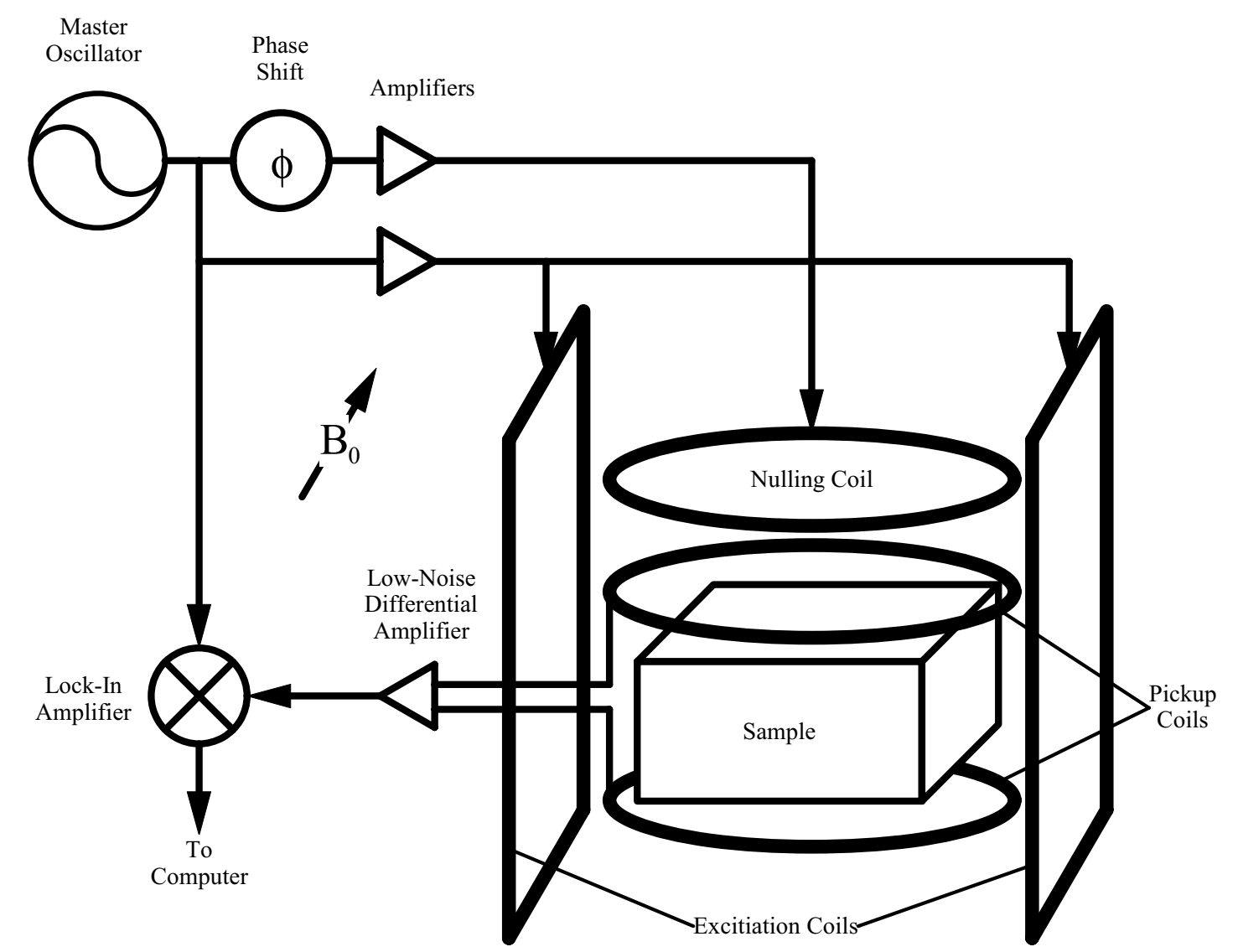

Figure 11 Schematic of AFP apparatus. The computer sweeps $B_{0}$ while reading the output of the lock-in amplifier.

We have obtained a signal from a water sample that was placed within a glass cell with a geometry that was very close to that of our helium test cell. No particular restrictions were placed on the purity of the water since a highly pure sample would have an unacceptably long $T_{1}$, requiring several seconds to thermally polarize. We considered whether to add a small amount of copper sulfate in order to further reduce the $T_{1}$, but found this unnecessary. This sample only provides a signal that is orders of magnitude below that of a 1-atmosphere ${ }^{3} \mathrm{He}$ sample even at modest polarization. The results of this measurement are shown in Figure 12, where the magnitude of the water signal is shown to be $\sim 6 \mu \mathrm{V}$. This measurement demonstrated that our apparatus was capable of detecting a signal from a 1 atmosphere ${ }^{3} \mathrm{He}$ cell at a polarization of only 2.2 parts per million. 


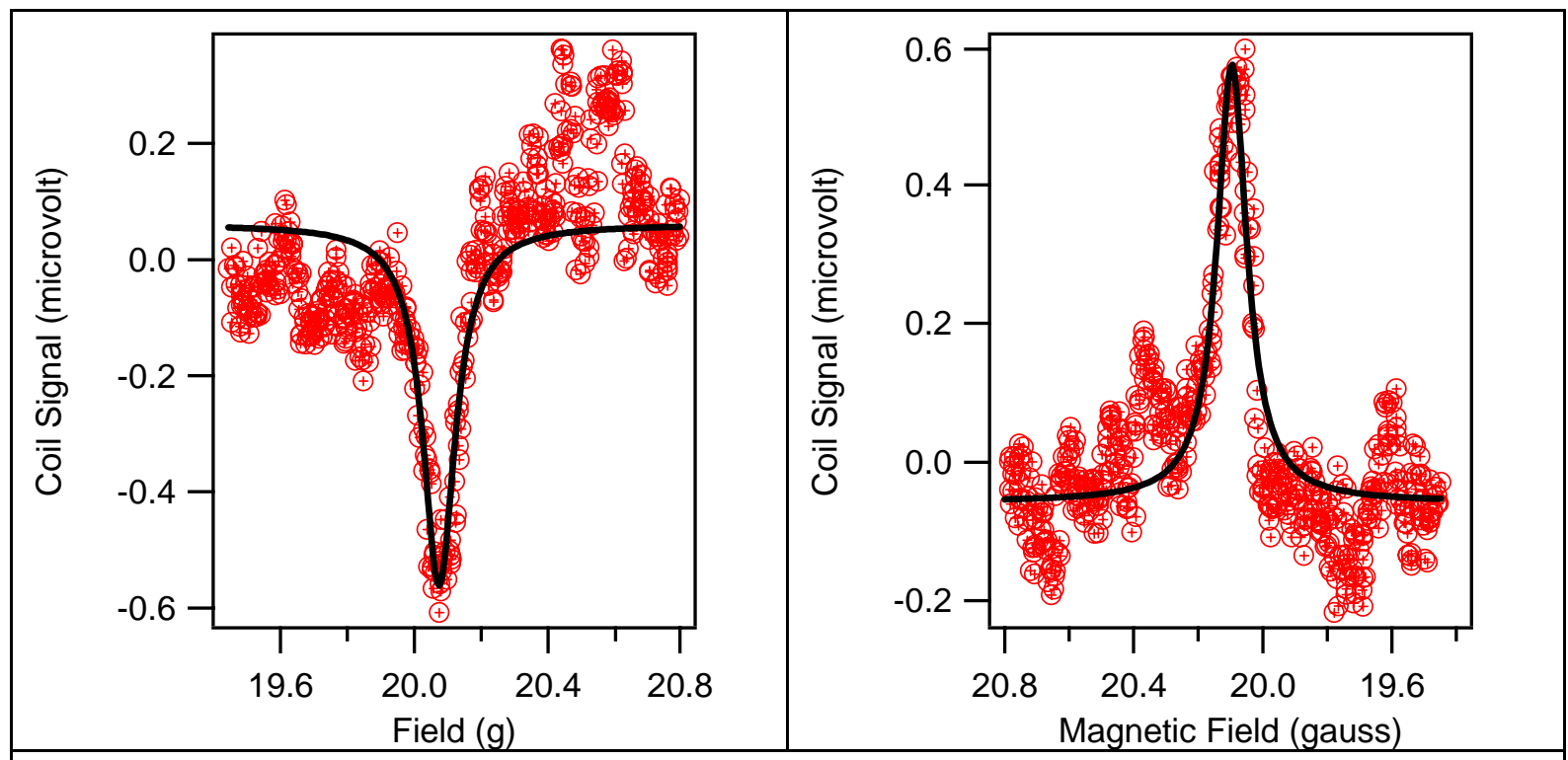

Figure 12 AFP Signal from a water sample. The resonant frequency was $87.8 \mathrm{kHz}$. The left-hand figure is swept up and the right-hand is the field swept down. The sweep time was 2.5 seconds in each direction (up and down). The data is the average of 10 sweeps. The fit shown is to a Lorentzian with FWHM 0.12 Gauss. This compares well to a measured excitation field strength of 0.11 gauss.

SRL has shown that it is possible to measure the polarization in the presence of the aluminum simply by placing this foil in the proper geometry such that it minimally blocks the NMR signal.

We have demonstrated that we can 1) measure even small AFP signals from partiallymetallic cells, 2) assemble cells with metallic components, and 3) make non-magnetic metallic valve components. We fell short of measuring the lifetime of aluminum cells and will have to leave this task to the Phase II project.

\section{Litterature Cited}

[1] B. Gabrys and O. Scharpf, "Scattering from polymers using polarised neutrons: a new development," Physica B, vol. 180, pp. 495-8, 1992.

[2] B. Gabrys, O. Scharpf, and D. G. Peiffer, "Short-range order in noncrystalline polymers examined with spin-polarized neutrons," Journal of Polymer Science, Part B (Polymer Physics), vol. 31, pp. 1891-5, 1993.

[3] H. B. Stuhrmann, N. Burkhardt, G. Dietrich, R. Juenemann, W. Meerwinck, M. Schmitt, J. Wadzack, R. Willumeit, J. Zhao, and K. H. Nierhaus, "Proton- and deuteron spin targets in biological structure research," Nuclear Instruments \& Methods in Physics Research, Section A (Accelerators, Spectrometers, Detectors and Associated Equipment), vol. 356, pp. 124-32, 1995.

[4] W. Knop, M. Hirai, H. J. Schink, H. B. Stuhrmann, R. Wagner, J. Zhao, O. Scharpf, R. R. Crichton, M. Krumpolc, K. H. Nierhaus, A. Rijllart, and T. O. Niinikoski, "A new polarized target for neutron scattering studies on biomolecules: first results from apoferritin and the deuterated 50S subunit of ribosomes," Journal of Applied Crystallography, vol. 25, pp. 2, 1992.

[5] W. Knop, H. B. Stuhrmann, R. Wagner, M. Wenkow-EsSouni, J. Zhao, O. Scharpf, M. Krumpolc, K. H. Nierhaus, T. O. Niinikoski, and A. Rijllart, "Polarized neutron scattering from dynamically polarized targets in biology," Nuclear Science and Engineering, vol. 110, pp. 316-29, 1992. 
[6] J. Zhao and H. B. Stuhrmann, "The in situ structure of the L3 and L4 proteins of the large subunit of E.coli ribosomes as determined by nuclear spin contrast variation," Journal de Physique IV (Colloque), vol. 3, pp. 233-6, 1993.

[7] H. Zabel, "Spin polarized neutron reflectivity of magnetic films and superlattices," Physica B, vol. 198, pp. 156-62, 1994.

[8] S. Itoh, Y. Endoh, S. Taketomi, and S. Chikazumi, "Determination of intraparticle structure of magnetite particles in a solvent by contrast variation method using polarized neutrons," Journal of Magnetism and Magnetic Materials, vol. 103, pp. 126-8, 1992.

[9] L. A. Axelrod, G. P. Gordeev, I. M. Lazebnik, V. T. Lebedev, S. V. Maleyev, A. I. Okorokov, V. V. Runov, V. N. Slyusar, B. P. Toperverg, A. D. Tretyakov, and R. Z. Yagud, "Small angle polarized neutron scattering in the superconducting Y-Ba-Cu-O ceramic," Materials Science Forum, vol. 27, pp. 27-28, 1988.

[10] S. K. Sinha, D. E. Moncton, D. C. Johnston, D. Vaknin, G. Shirane, and C. Stassis, "Antiferromagnetism in the high-T/sub c/ related compounds," Journal of Applied Physics, vol. 63, pp. 4015-18, 1988.

[11] T. R. Gentile, G. L. Jones, A. K. Thompson, J. Barker, C. Glinka, B. Hammouda, and J. Lynn, "Polarization Analysus and the NG3 SANS Apparatus, Separation of coherent and spin-incoherent scattering," 1999.

[12] O. Shaerpf, "Thin film devices and their role in future neutron spectroscopic investigations," Physica B, vol. 174, pp. 514-527, 1991.

[13] C. F. Majkrzak, "Advances in polarized neutron reflectometry," Physica B, vol. 213, pp. 213-214, 1995.

[14] K. P. Coulter, T. E. Chupp, A. B. McDonald, C. D. Bowman, J. D. Bowman, J. J. Syzmanski, V. Yuan, G. D. Cates, D. R. Benton, and E. E. Earle, "Neutron Polarization with a polarized ${ }^{3} \mathrm{He}$ spin filter," Nucl. Instr. and Meth., vol. A288, pp. 463, 1990.

[15] W. Heil, K. Andersen, D. Hofmann, H. Humblot, J. Kulda, E. Lelievre-Berna, O. Schaerpf, and F. Tasset, "33e neutron spin filter at ILL," Physica B, vol. 241, pp. 241-243, 1997.

[16] J. D. Bowman, C. M. Frankle, A. A. Green, J. N. Knudson, S. I. Penttila, S. J. Seestrom, Y. YiFen, V. W. Yuan, B. E. Crawford, N. R. Roberson, C. R. Gould, D. G. Haase, L. Y. Lowie, G. E. Mitchell, S. I. Stephenson, P. P. J. Delheij, E. I. Sharapov, H. Postma, Y. Masuda, H. M. Shimizu, M. Iinuma, A. Masaike, Y. Matsuda, and K. Fukuda, "Parity violation in the compound nucleus," Chinese Journal of Physics, vol. 32, pp. 989-1002, 1994.

[17] T. Prokscha, "Test und Weiterentwicklung eines mechanisch komprimierten, polarisierten 3HeTargets," Universitat Mainz, 1991.

[18] R. F. C. Farrow, R. F. Marks, M. F. Toney, S. David, A. J. Kellock, J. A. Borchers, K. V. O'Donovan, and D. J. Smith, "Spontaneous chemical ordering and exchange bias in epitaxial $\mathrm{Mn} / \mathrm{sub}$ 0.52/Pd/sub 0.48//Fe(001) bilayers prepared at room temperature," Applied Physics Letters, vol. 80, pp. 808-10, 2002.

[19] V. F. Sears, "Thermal-Neutron Scattering Lengths and Cross Sections for Condensed-Matter Research," Chalk River Nuclear Laboratories, Chalk River, Ontario, Canada Publication AECL7980, 1984.

[20] G. L. Greene, A. K. Thompson, and M. S. Dewey, "A method for the accurate determination of the polarization of a neutron beam using a polarized /sup 3/He spin filter," Nuclear Instruments \& Methods in Physics Research, Section A (Accelerators, Spectrometers, Detectors and Associated Equipment), vol. 356, pp. 177-80, 1995.

[21] W.Heil, K. H. Andersen, R. Cywinski, H. Humblot, C. Ritter, T. W. Roberts, and J. R. Stewart, "Large solid-angle polarisation analysis at thermal neutron wavelengths using a ${ }^{3} \mathrm{He}$ spin filter," Nucl. Instrum. and Meth. in Phys. Res. A, vol. 485, pp. 551 -570, 2002.

[22] L. D. Cussen, D. J. Goossens, and T. J. Hicks, "3He neutron polarising filters- theoretical comparison with supermirrors and heusler alloy polarisers," Nucl. Instrum. and Meth. in Phys. Res. A, vol. 440, pp. 409-420, 2000.

[23] B. Driehuys, G. D. Cates, and W. Happer, "Surface relaxation mechanisms of laser-polarized /sup 129/Xe," Physical Review Letters, vol. 74, pp. 4943-6, 1995. 
[24] W. Heil, H. Humblot, E. Otten, M. Schafer, R. Sarkau, and M. Leduc, "Very long nuclear relaxation times of spin polarized helium 3 in metal coated cells," Physics Letters A, vol. 201, pp. 337-43, 1995.

[25] R. S. Timsit, J. M. Daniels, and A. D. May, "Nuclear relaxation of ${ }^{3}$ he gas on various solid surfaces," Canadian Journal of Physics, vol. 49, pp. 560-75, 1971.

[26] J. F. O'Hanlon, A Users Guide to Vacuum Technology, 2nd ed. New York: John Wiley \& Sons, 1989.

[27] R. W. Mair, G. P. Wong, D. Hoffmann, M. D. Hürlimann, S. Patz, L. M. Schwartz, and R. L. Walsworth, "Probing Porous Media with Gas Diffusion NMR," Phys. Rev. Lett., vol. 83, pp. 33243327, 1999.

[28] $\quad$ F. D. Colegrove, L. D. Shearer, and G. K. Walters, "Polarization of 3He gas by optical pumping," Phys. Rev., vol. 132, pp. 2561, 1963.

[29] J. Becker, W. Heil, B. Krug, M. Leduc, M. Meyerhoff, P. J. Nacher, E. W. Otten, T. Prokscha, L. D. Schearer, and R. Surkau, "Study of mechanical compression of spin-polarized /sup 3/He gas," Nuclear Instruments \& Methods in Physics Research, Section A (Accelerators, Spectrometers, Detectors and Associated Equipment), vol. 346, pp. 45-51, 1994.

[30] M. Ebert, T. Grossmann, W. Heil, W. E. Otten, R. Surkau, M. Leduc, P. Bachert, M. V. Knopp, L. R. Schad, and M. Thelen, "Nuclear magnetic resonance imaging with hyperpolarised helium-3," Lancet, vol. 347, pp. 1297-9, 1996.

[31] H. Middleton, R. D. Black, B. Saam, G. D. Cates, G. P. Cofer, R. Guenther, W. Happer, L. W. Hedlund, G. A. Johnson, K. Juvan, and et al., "MR imaging with hyperpolarized 3He gas," Magn Reson Med, vol. 33, pp. 271-5, 1995.

[32] T. Beardsley, "Seeing the Breath of Life," in Scientific American, 1999, pp. 33-34.

[33] M. S. Rosen, T. E. Chupp, K. P. Coulter, R. C. Welsh, and S. D. Swanson, "Polarized ${ }^{129}$ Xe optical pumping/spin exchange and delivery system for magnetic resonance spectroscopy and imaging studies," Review of Scientific Instruments, vol. 70, 1999.

[34] M. E. Wagshul and T. E. Chupp, "Laser optical pumping of high-density Rb in polarized /sup 3/He targets," Physical Review A (Atomic, Molecular, and Optical Physics), vol. 49, pp. 3854-69, 1994.

[35] C. D. Keith, T. C. Black, X. Fei, M. Flamini, T. R. Gentile, G. L. Jones, D. R. Rich, W. M. Snow, A. K. Thompson, and F. E. Wietfeldt, "Neutron polarizers based on polarized ${ }^{3} \mathrm{He}, "$ Nucl. Instrum. Methods Phys. Res. A, Accel. Spectrom. Detect. Assoc. Equip., vol. 402, pp. 236-240, 1998.

[36] R. Surkau, J. Becker, M. Ebert, T. Grossmann, W. Heil, D. Hofmann, H. Humblot, M. Leduc, E. W. Otten, D. Rohe, K. Siemensmeyer, M. Steiner, F. Tasset, and N. Trautmann, "Realization of a broad band neutron spin filter with compressed, polarized /sup 3/He gas," Nuclear Instruments \& Methods in Physics Research, Section A (Accelerators, Spectrometers, Detectors and Associated Equipment), vol. 384, pp. 444-50, 1997.

[37] F. Tasset, T. E. Chupp, J. P. Pique, A. Steinhof, A. Thompson, and E. Wasserman, "First test at ILL of a gaseous /sup 3/He neutron polarization filter," Physica B, vol. 180, pp. 896-8, 1992.

[38] F. Tasset, "Towards helium-3 neutron polarizers," Physica B, vol. 213, pp. 213-214, 1995.

[39] G. Eckert, W. Heil, M. Myerhoff, E. W. Otten, R. Surkau, M. Werner, M. Leduc, P. J. Nacher, and L. D. Schearer, "A Dense Polarized ${ }^{3} \mathrm{He}$ Target based on Compression of Optically Pumped Gas," Nucl. Inst. and Meth. in Phys. Res., vol. A320, pp. 53-65, 1992.

[40] O. Shaerpf, "Comparison of theoretical and experimental behaviour of supermirrors and discussion of limitations," Physica B, vol. 156 and 157, pp. 631-638, 1989.

[41] O. Shaerpf, "Properties of beam-bender type neutron polarizers using supermirrors," Physica B, vol. 156 and 157, pp. 639-646, 1989.

[42] M. S. Albert, G. D. Cates, B. Driehuys, W. Happer, B. Saam, C. S. Springer, Jr., and A. Wishnia, "Biological magnetic resonance imaging using laser-polarized /sup 129/Xe," Nature, vol. 370, pp. 199-201, 1994. 